\title{
Sensores Virtuais para Detecção de Descargas Parciais em Transformadores de Potência
}

\author{
Brendo S. Barbosa* Pyramo P. Costa* Rose M. S. Batalha* \\ Daniel F. Leite ${ }^{* *}$ \\ * Programa Pós-Graduação em Engenharia Elétrica, Pontíficia \\ Universidade Católica de Minas Gerais, PUC-MG, Brasil \\ (brendo.s.barbosa@gmail,pyramo@pucminas.br, \\ batalha@pucminas.br). \\ ** Departamento de Automática, Universidade Federal de Lavras, \\ UFLA, Brasil (daniel.leite@ufla.br).
}

\begin{abstract}
Partial discharge is a common type of fault in power transformers. It indicates the state of the insulation material. Identifying their existence is not an easy task. Some techniques such as chromatographic analysis of the refreshing oil have been used for offline detection of partial discharges. In this paper we present a Virtual Sensor scheme for online partial-discharge detection. The virtual sensor consists of two steps. First, the pre-processing step consists in cleaning the data, and applying wavelet transforms. Second, a neural classifier, namely, a radialbasis-function neural network (RBF), an adaptive neuro-fuzzy system (ANFIS) or an evolving neuro-fuzzy system (DENFIS) is employed. In this paper partial discharges are simulated using a lumped-parameter model. For performance analysis, disturbances are considered. System results have shown to be feasible and robust. The use of virtual sensors for online detection of partial discharges is encouraging.

Resumo: Descarga parcial é um tipo de falta comum em transformadores de potência. Ela indica o estado do material isolante dos equipamentos e sua identificação não é uma tarefa fácil. Algumas técnicas como análise cromatográfica do óleo refrigerante têm sido utilizadas na detecção das descargas parciais off-line. Neste artigo é apresentada uma proposta de Sensor Virtual para detecção de descargas parciais on-line. O sensor virtual é constituído de duas seções. Primeiro, o pré-processamento, onde os dados de correntes do transformador são tratados e transformadas wavelet são empregadas. Segundo, o classificador inteligente, constituído por uma rede neural de base radial (RBF), um sistema neuro-fuzzy adaptativo (ANFIS) ou um sistema neuro-fuzzy evolutivo (DENFIS). Neste artigo as descargas parciais foram simuladas utilizando um modelo de parâmetros concentrados. Para análise de desempenho são consideradas situações de distúrbio. Os resultados obtidos mostram-se viáveis, assim como a utilização de sensores virtuais para detecção on-line de descargas parciais.
\end{abstract}

Keywords: Neural Network, Power Transformer, Partial Discharge Detection, Virtual Sensors. Palavras-chaves: Rede Neural, Transformador, Detecção de Descarga Parcial, Sensores Virtuais.

\section{INTRODUÇÃO}

A descarga parcial (Partial Discharge, PD) é uma das faltas mais comuns em equipamentos elétricos de potência. Elas são causadas devido à modificações na estrutura do material isolante empregado no enrolamento do equipamento, o que pode gerar bolhas de ar e perda das características dielétricas em consequência ao envelhecimento precoce. O material isolante não suporta o gradiente de tensão imposto sem que ocorra a formação de pequenos arcos elétricos internos nos enrolamentos.

As ocorrências de PD podem gerar a perda da estabilidade dielétrica de um sistema de isolamento. Isso se deve à tendência de redução da capacidade dielétrica do isolante, que se estende até a formação de uma descarga plena (Zhu et al., 2015). Há também a possibilidade da formação de gases inflamáveis se as descargas ocorrerem em contato com o óleo refrigerante utilizado nos equipamentos.

Atualmente, a análise cromatográfica do óleo refrigerante do transformador é muito utilizada para a indicação de falta nesses equipamentos. Isso se dá pela sua capacidade de identificar múltiplos tipos de faltas, como mostrado em (Golarz, 2016). Cada tipo de falta produz combinações específicas de gases, que se acumulam no óleo. A interseção de gases para diferentes faltas e existência de várias faltas simultâneas torna gera uma certa imprecisão ao método, como relatado em IEEEC57.104-2008 (2008). Além disso, há um custo operacional e logístico envolvido em realizar 
a coleta e o transporte de amostras de óleo de cada equipamento de subestações de uma ampla região para os centros de análise química. Sendo assim, a análise de gases dissolvidos não é uma metodologia aceitável para os novos conceitos de redes elétricas inteligentes (smart grids (SG)) (Colak et al., 2014).

Khramshin et al. (2015) cita os desafios para implementação de SG dando ênfase aos transformadores de potência e às mudanças necessárias para o novo cenário da rede. Dentre essas mudanças incluem o controle contínuo das condições técnicas de operação dos transformadores, detecção precoce e localização de falha, e a programação eficaz dos trabalhos de manutenção. Tais itens não podem ser atendidos pelos modelos de operação e, principalmente, de manutenção utilizados atualmente.

Muito se discute sobre formas mais eficazes para a detecção de faltas que podem acometer os equipamentos elétricos de potência, incluindo as PD. A necessidade de detectar e medir a intensidade e a localização de descargas parciais é de fundamental importância em programas de manutenção. Porém, como visto em (Zhenquan et al., 2009), sua detecção e identificação ainda é muito complicada. Ademais, a maioria dos métodos de detecção propostos, conforme IEEEstd1434-2014 (2014), são métodos off-line.

Trabalhos como (Chan et al., 2015) e (Wan and Chan, 2016) abordam alguns métodos on-line propostos pelo IEEE-Std-1434. São utilizadas ondas sonoras ou eletromagnéticas emitidas no momento da centelha elétrica da descarga para indicar sua presença e encontrar sua localização. Alguns trabalhos partem para o uso de grandezas elétricas geradas pelas PD como em (Mohamed and Lewin, 2009) e (Jeyabalan and Usa, 2009). Neste trabalhos são utilizados circuitos equivalentes de parâmetros concentrados para a simulação dos enrolamentos de um transformador de potência submetidos à descargas parciais. Yini and Lixing (2007) utilizam uma rede neural Hopfield e Mondal and Kumbhar (2017) utilizam um método baseado em ortogonalização de Gram Schmidt para realizarem detecção e localização de PD.

Visando uma solução que detecte as PD atendendo as condições impostas por Khramshin, e sem que haja a necessidade da adição de novos sensores no transformador, esse trabalho propõe o uso de Sensores Virtuais (SV) não físicos e indiretos. O sistema de monitoramento é capaz de inferir uma variável não medida a partir de um modelo de correlação das variáveis medidas. A sua aplicação torna-se útil em situações em que o sensor para uma determinada variável é muito caro ou deve atuar em um ambiente improprio à sua instalação. Algumas vezes, o sensor físico simplesmente não existe (Shao et al., 2015).

Exemplos de SV podem ser vistos em (Ascencio, 2000). É proposta estimação inferencial para se obter a medição da biomassa a partir de diversas variáveis como dióxido de carbono, pH, e oxigênio. No trabalho de Lin et al. (2006), é utilizado um SV para mensurar a quantidade de Cal livre e NOx produzidos dentro de um forno de produção de cimento. Em (Lotufo and Garcia, 2008) foi desenvolvido um SV para monitorar a cota de nitrogênio interno de células de fitoplâncton. Pode ser notado que a maioria dos trabalhos são voltados para a área da engenharia química.
Este trabalho apresenta uma nova proposta de desenvolvimento de um SV para detecção de descargas parciais em transformadores de potência. O SV deve ser robusto às condições de entradas uma vez que os sinais de sensores físicos instalados podem não serem íntegros. A metodologia proposta pode ser aplicada a outros equipamentos elétricos de potência, criando uma condição on-line de predição do estado dos equipamentos e de faltas incipientes ou catastróficas. O sistema é empregável às novas realidades impostas por redes inteligentes oferecendo segurança, robustez e maior confiabilidade à rede.

\section{REFERENCIAL TEÓRICO}

\subsection{Sensor Virtual}

Os sensores virtuais são softwares (potencialmente embarcados) que trabalham com a correlação dos dados dos sensores presentes em uma planta para inferir uma variável não medida. Sua vantagem é a dispensa da existência de um dispositivo físico. O sensor virtual é modular à diferentes tipos de correlações e dados de entrada. Um SV é constituído de duas etapas: o pré-processamento é a etapa em que ocorre a extração de características; em seguida, as características obtidas alimentam um modelo inteligente. Em ambas as etapas, existe a preocupação quanto ao uso da ferramenta matemática mais adequada para a situação onde o sensor está aplicado.

Os dados coletados durante a operação do processo geralmente contêm imprecisões. Estas podem ocorrer devido ao resultado de diferentes distúrbios, degradação, além de não haver garantia da integridade dos dados oferecido pelos sensores. Tais situações são identificadas e estudadas neste trabalho para validar a robustez do SV proposto. Para remover tais imprecisões que afetam negativamente a qualidade do modelo, o pré-processamento de dados é fundamental (Lin et al., 2006) (Lucas et al., 2018).

SV são frequentemente tratados por métodos estatísticos para extração de características (Dixon, 1999; Lin et al., 2006). Tais métodos incluem: análise de componentes principais (PCA), mínimos quadrados parciais (PLS), máquina de vetor suporte (SVM), métodos de regressão, wavelets, métodos de clusterização como K-Means, filtro de Kalman, dentre outros (Kadlec et al., 2009) (Kadlec et al., 2010) (Silva et al., 2018a) (Silva et al., 2018b). Tais métodos também podem ser usados como o modelo de correlação ou para o tratamento de outliers.

O modelo inteligente, que faz o papel de sensor virtual em si, pode ser do tipo caixa branca, cinza ou preta, dependendo principalmente do tipo de correlação existente entre as variáveis medidas e a variável desejada. Para correlações lineares, ferramentas como regressão linear por mínimos quadrados ou os métodos supracitados são soluções geralmente eficazes. Contudo, para relações não-lineares e variantes no tempo (não-estacionárias), as melhores opções são ferramentas neuro-fuzzy, principalmente sistemas evolutivos (Kasabov, 2007) (Leite et al., 2013). Um artigo Survey recente resume o estado da arte da área de sistemas fuzzy e neuro-fuzzy evolutivos (Skrjanc et al., 2019).

\subsection{DENFIS}

Sistemas Conexionistas Evolutivos (Evolving Connectionist Systems - ECoSs) (Watts, 2009) são uma família 
de algoritmos construtivos de rede neural que foram propostos originalmente em (Kasabov, 1998). Um ECoS é um sistema adaptativo e incremental de aprendizagem e conhecimento que evolui sua estrutura e funcionalidade. No núcleo do sistema está uma arquitetura conexionista que consiste em neurônios (unidades de processamento de informações) e conexões entre neurônios. Estas utilizam algoritmos incrementais de aprendizagem que operam de forma contínua no tempo, e adaptam sua estrutura e funcionalidade através da interação contínua com o meio ambiente e com outros sistemas. Dentre tais redes destacase o modelo DENFIS (Dynamic Evolving Neural-Fuzzy Inference System). Uma aplicação dos princípios de ECoS à uma RNA (Rede Neural Artificial) que implementa um sistema de inferência fuzzy Takagi-Sugeno é mostrada em (Kasabov, 2002).

O DENFIS utiliza o método chamado ECM (Evolving Clustering Method), que se baseia no conceito de adicionar e modificar dinamicamente os clusters à medida que novos dados são apresentados. Ele tem apenas um parâmetro a ser definido, o limite de distância $D_{t h r}$. Quando novos clusters são adicionados, seu raio $R$ é definido como zero, e seu centro é igual ao vetor de entrada $I_{n}$. Conforme exista a ocorrência de vetores próximo à um cluster, seu raio $R$ é aumentado, e seu centro é deslocado em direção à amostra. Porém, o raio $R$ não pode exceder o limite, $D_{t h r}$. $\mathrm{O}$ algoritmo ECM é mostrado na Fig. 1.

Quando o cluster a é atualizado, seu centro é deslocado para mais perto de $I_{n}$ e seu raio $R_{a}(t+1)$ é definido conforme

$$
R_{a}(t+1)=\frac{S_{i, a}}{2}
$$

em que o novo centro de $a, C_{a}(t+1)$ é ajustado de modo que sua distância esteja na reta entre $C_{a}(t)$ e $I_{n}$, à uma distância de $R_{a}(t+1)$.

Embora o ECM pareça ser um método de clusterização útil em si, sua função principal é produzir os termos antecedentes de um sistema de inferência fuzzy. Os antecedentes são formulados pela combinação dos clusters mais ativos por um vetor de entrada. As funções lineares consequentes de uma regra fuzzy são adaptadas usando o método LSE ( $L i$ near Least-Square Estimator) sobre os centros dos cluster existentes. Assim, cada cluster é usado como um envólucro dos dados de entrada e como uma região onde a função linear consequente é válida. Para qualquer vetor de entrada $I$, a saída do modelo DENFIS é calculada como a média ponderada das contribuições locais das regras ativas.

\section{METODOLOGIA}

O fluxograma geral do esquema de detecção utilizando o sensor virtual é mostrado na Fig. 2. Fundamentalmente, o fluxograma consiste nos três elementos citados: (1) a simulação das PD internas no enrolamento do transformador utilizando o modelo de parâmetros concentrados; (2) o pré-processamento e extração de características dos dados; e (3) a rede neural para captura da correlação entre as características e faltas.
Create the first cluster centre $C_{0}$ from the first example $\mathbf{I}_{0}$

for each subsequent vector $\mathbf{I}_{n}$ do

Find the minimum distance $D_{\min }$ between $\mathbf{I}_{n}$ and each cluster centre $C_{n}$

if $D_{\min }$ is less than any cluster radius then

Add $\mathbf{I}_{n}$ to the nearest cluster else

Find the cluster $a$ with minimum value of $S_{i, j}$, where $S_{i, j}=D_{i, j}+R_{i}, D_{i, j}$ is the distance between the cluster centre and vector $j$, and $R_{i}$ is the radius of cluster $i$

$$
\begin{aligned}
& \text { else } \\
& \text { Update } a \\
& \text { end if } \\
& \text { end if }
\end{aligned}
$$$$
\text { if } S_{i, a}>2 D_{t h r} \text { then }
$$$$
\text { Create a new cluster }
$$$$
\text { end for }
$$

Figura 1. Algoritmo de Treinamento ECM

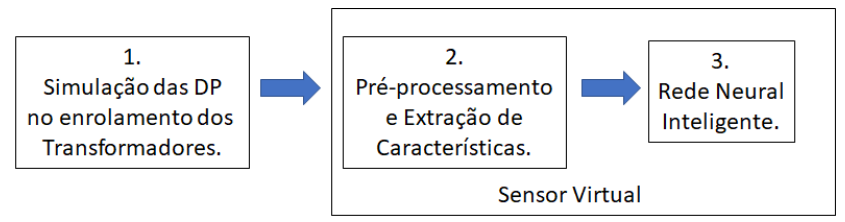

Figura 2. Fluxograma do esquema de detecção de falta utilizando Sensor Virtual

\subsection{Simulação}

As PD foram simuladas a partir do ambiente Simulink com o circuito equivalente de parâmetros concentrados do enrolamento de alta tensão de um transformador de $1 M V A$ e $6,6 k V$ em condição nominal. Essa escolha foi feita já que a relação pulso/corrente é maior em relação ao enrolamento de baixa tensão. A representação do circuito pode ser vista na Fig. 3. Cada porção do circuito corresponde a um segmento do enrolamento do transformador. Os valores utilizados são $L=220 \mathrm{mH}, C_{2}=2500 p F, C_{1}=450 p F$ e $R=10 \Omega$, vide (Akbari et al., 2002).

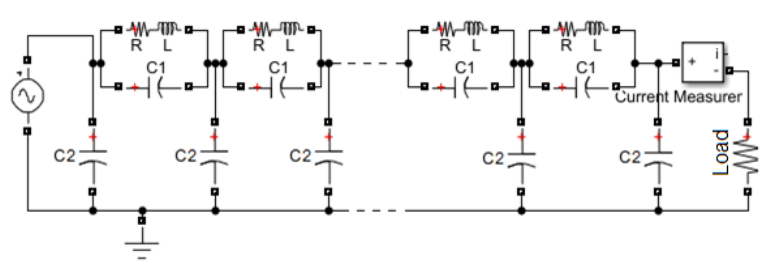

Figura 3. Circuito Equivalente de Parâmetros Concentrados

Pulsos são inseridos em um determinado segmento, simulando as caracterisitcas das PD internas. Isso é feito a partir da inserção do pulso em uma dada posição da forma de onda, como pode ser visto em (IEEEC57.1132015,2015 ). Foram simulados 0,667 segundos (um total de 40 ciclos de onda). Os 10 primeiros ciclos possuíam PD. Os 10 ciclos seguintes não possiam PD, e assim por diante.

Os dados foram janelados em dois ciclos de onda. Cada ciclo possui 1667 pontos além de serem criadas três situações 
distindas para a avaliação da robustez do sensor virtual. São elas: (1) nenhuma interferência; (2) substituição aleatória de $15 \%$ dos dados por outliers; e (3) deleção aleatória de $15 \%$ dos dados.

\subsection{Pré-Processamento}

O pré-processamento é subdividido em duas etapas. A primeira delas é a detecção e retirada de outliers, e o preenchimento dos dados faltantes. Nesse sentido são feitas duas regressões senoidais, sendo a primeira com a presença de outliers, em que os dados que estiverem além de um limiar são removidos, conforme:

$$
\left.\mid X_{n}-R E_{x}\left(x_{n}\right)\right) \mid>L_{t h}
$$

onde $x_{n}$ é a entrada no instante $n, R E_{x}\left(x_{n}\right)$ é a regressão da janela no ponto $x_{n} ; n$ varia de 1 até 1667 , e $L_{t h}$ é o limiar. Em funções lineares é utilizado a regra $3 \sigma$, i.e., o método de Hampel.

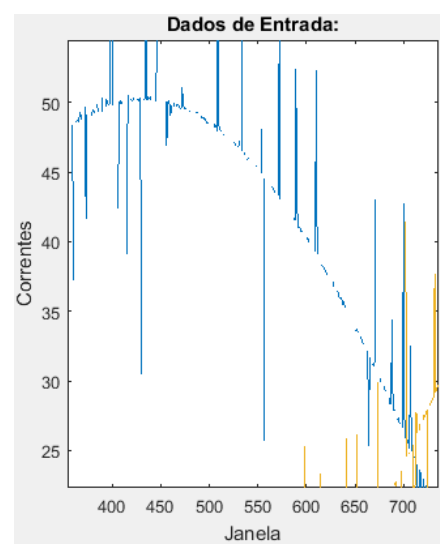

(a) Entrada com Disturbios

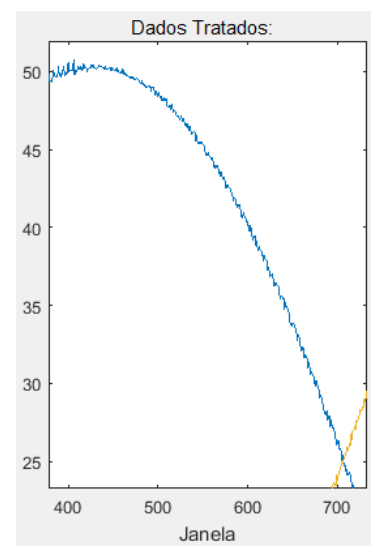

(b) Entrada Suavizada
Figura 4. Pré-Processamento dos Dados

Na segunda regressão os dados retirados na etapa anterior bem como os dados ausentes são substituídos conforme visto na Fig. 4. Isso é feito para que os outliers não criem nenhuma tendência na regressão utilizada para substituilos.

A segunda etapa do pré-processamento consiste na transformada wavelet e cálculo do espectro de energia. Diferentes transformadas wavelets, daubechie 2 a 8 (db2, db8) e symlet 2 a 8 (sym2, sym8) foram utilizadas para a extração de característica da janela pré filtra. A Wavelet cria uma desmenbramento do sinal de uma janela em vários níveis onde os sinais de alta frequência da janela são seprados como coeficientes de detelhe de $1^{\circ}$ nível e o restante fica como coeficiente de aproximação. Separando os sinais de alta frequência dos coefientes de aproximação de $1^{\circ}$ nível, tem-se os coeficientes de detelhe de $2^{\circ}$ nível. Desse forma é possivel criar uma análise multiresolução da janela onde no trabalho são utilizados os coeficientes de detalhe de segundo e terceiro níveis, visto que o ruído branco danifica a decomposição em primeiro nível.

Na sequência é feito o cálculo do espectro de energia dos coeficientes de detalhe resultantes obtidos sobre todo o intervalo da janela trabalhada. A energia de um sinal é o somatório de quadrado da função do sinal, como pode ser visto na Equação 3.

$$
E\left(T W D, D_{i}\right)=\sum_{l=1}^{N} X_{l}^{2}
$$

onde $X$ é a amplitude dos coeficientes wavelets obtidos a partir das amostras contidas na janela de tempo.

Por fim é feita a normalização da janela com uso do fator de escala, para não criar nenhum viés por diferença de amplitude dos vetores de entrada que é muito nociva às redes neurais artificiais utilizadas a seguir.

\subsection{Redes Neurais}

Foram utilizadas as redes neurais artificiais de base-radial RBF, rede neuro-fuzzy ANFIS e a rede neuro-fuzzy evolutiva DENFIS a fim de criar um modelo de correlação de dados. Elas funcionam como classificadores e indicam a presença ou não das PD bem como sua localização. Para não ocorrer tendências de validação positiva foi utilizado o método de validação cruzada 5 -fold. Todas as combinações de avaliação da robustez do sensor, extração de características com diferentes famílias wavelets e redes neurais artificiais são empregadas e comparadas com relação à eficiência e robustez.

\section{ANÁLISE DOS RESULTADOS}

Diferentes redes neurais, RBF, ANFIS e DENFIS são utilizadas com diferentes transformadas wavelets, daubechie e symlets para cada situação de entrada, podendo ser sem interferência, com $15 \%$ de outliers e com $15 \%$ dos dados ausentes. Os resultados são comparados pela eficiência, que é uma ferramenta estatística poderosa para comparações de classificadores, dada pela média da sensibilidade e da especificidade obtidas na matriz de confusão.

A sensibilidade é dada pela proporção de verdadeiros positivos, significando a capacidade do sistema em predizer corretamente a condição positiva para os casos positivos.

$$
\text { Sensibilidade }=\frac{\text { Identificado Positivo }}{\text { Total de Positivo }}
$$

A especificidade é dada pela proporção de verdadeiros negativos, significando a capacidade do sistema em predizer corretamente a ausência da condição para casos negativos.

$$
\text { Especificidade }=\frac{\text { Identificado Negativo }}{\text { Total de Negativos }}
$$

Calculadas as eficiências para cada situação explicada acima temos os resultados expostos nos gráficos radar, sendo este um método que apresenta dados multivariáveis na forma de um gráfico polar utilizando raios equipotenciais para referência da amplitude das variáveis de um determinado modelo, onde cada modelo fica representado em um ângulo diferente. Desta forma, o gráfico permite realizar a comparação de muitos modelos sob a mesma escala de grandeza. Nas Fig. 5, Fig. 6 e Fig. 7 é possível ver os resultados obtidos para as condições de ser sem 
interferência, com $15 \%$ de outliers e com $15 \%$ dos dados ausentes respectivamente.

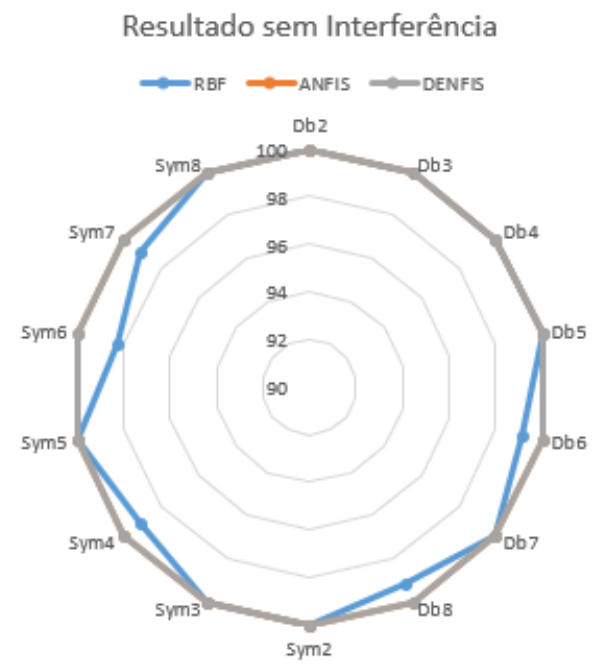

Figura 5. Resultados sem distúrbios

Pode-se ver na Fig. 5 que todas as redes neurais obtiveram bons resultados para identificar a presença das descargas parciais na situação ideal. Aqui percebe-se também que houve uma sobreposição dos resultados entre os classificadores DENFIS e ANFIS.

\section{Resultados com $15 \%$ de Outliers}

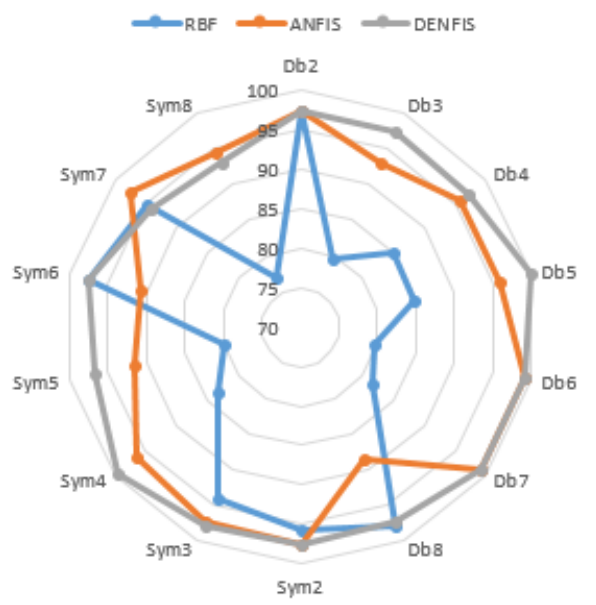

Figura 6. Resultados com $15 \%$ de outliers

Na Fig. 6 é visto o resultado obtido com adição $15 \%$ de outliers, onde podemos observar que o DENFIS e o ANFIS obtiveram boas eficiências e o RBF com resultado inferior comparada às outras. Com a retirada de $15 \%$ dos dados de entrada, vistos na Fig. 7, as redes ANFIS e DENFIS conseguiram boas eficiências para todas as transformadas. Isso não pode ser afirmado da rede RBF, que obteve uma queda acentuada de eficiência com a ausência de dados, se tornando uma rede não adequada para trabalhar em ambientes onde o sensor será submetido a este tipo de situação.

Comparando as Figs. 6 e 7 pode-se perceber que as redes ANFIS e DENFIS, apesar de terem obtido bons resultados, se tornaram menos eficientes à presença de outliers. Isso
Resultado com $15 \%$ de dados Ausentes

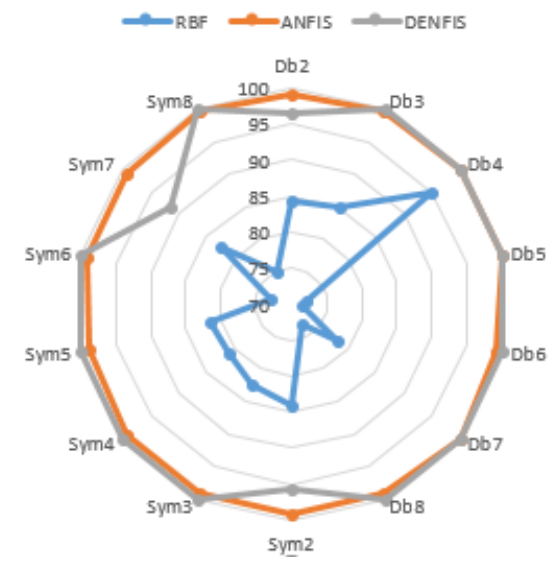

Figura 7. Resultados com $15 \%$ de dados faltantes

pode ocorrer devido aos outliers sobreviventes a primeira camada do pré-processamento prejudicarem as transformadas wavelets. Já para a RBF é percebida a piora de desempenho com a adição dos dois distúrbios.

\section{CONCLUSÃO}

Este artigo apresentou um modelo de um sensor virtual para a detecção de descargas parciais em transformadores de potência. O sensor foi implementado a partir de diferentes classificadores neurais, diferentes famílias de transformadas wavelet e uma metodologia de retirada de outliers e preenchimento de dados ausentes utilizando sinais provenientes de um circuito de parâmetros concentrados e simulações de um transformador sob influência de PD internas.

Foi demonstrado que a metodologia de SV proposta é eficiente para a detecção da existência das PD. O modelo que apresentou o melhor resultado médio para os diferentes tipos de entrada foi o modelo neuro-fuzzy evolutivo DENFIS seguido por ANFIS. O modelo RBF apresentou piores resultados quando comparado aos demais quanto a adição de distúrbios.

Percebe-se também que os classificadores DENFIS e ANFIS foram robustos, apresentando uma pequena queda de eficiência com o acréscimo dos distúrbios, possuindo resultados consistentes para praticamente todas as famílias wavelet. Já o classificador RBF possui resultados consistentes para apenas algumas famílias wavelet na situação de adição de outliers.

Em trabalhos futuros, outros métodos matemáticos como outras transformadas ou diferentes métodos de detecção de outliers bem como a adição de distúrbios de natureza elétrica que ocorrem nas subestações onde os transformadores de potência são instalados devem ser considerados, por exemplo o chaveamento de capacitores e mudanças de tap do transformador. Pode-se também estender a metodologia proposta para a localização de ocorrência da PD bem como a identificação de seu tipo, tais como PD na corona, entre fases e no aterramento. Há também a possibilidade de implementação de vários sensores virtuais em um mesmo hardware. 


\section{AGRADECIMENTOS}

O último autor agradece o apoio do Instituto Serrapilheira, número do processo Serra - 1812-26777, e à Fundação de Amparo à Pesquisa do Estado de Minas Gerais (FAPEMIG), processo APQ-03384-18.

\section{REFERÊNCIAS}

Akbari, A., Werle, P., Borsi, H., and Gockenbach, E. (2002). Transfer function-based partial discharge localization in power transformers: A feasibility study. IEEE Electrical Insulation Magazine, 18(5), -.

Ascencio, L. (2000). Biomass estimation using artificial neural networks on field programmable analog devices. IEEE International Symposium, 1, -.

Chan, J.C., Ma, H., and Saha, T.K. (2015). Timefrequency sparsity map on automatic partial discharge sources separation for power transformer condition assessment. IEEE Transactions on Dielectrics and Electrical Insulation, 22(4), 2271-2283.

Colak, I., ErsanKabalci, GianlucaFulli, and StavrosLazarou (2014). A survey on the contributions of power electronics to smart grid systems. Renewable and Sustainable Energy Reviews, 47, 562-579.

Dixon, K. (1999). Rave: A real and virtual environment for multiple robot systems. IEEE/RSJ International Conference on Intelligent Robots and Systems, -.

Golarz, J. (2016). Understanding dissolved gas analysis (dga) techniques and interpretations. Transmission and Distribution Conference and Exposition, - .

IEEEC57.104-2008 (2008). IEEE Guide for the Interpretation of Gases Generated in Oil-Immersed Transformers. IEEE Standard.

IEEEC57.113-2015 (2015). Recommended Practice for Partial Discharge Measurement is Liguid-Filled Power Transformers and Shunt Reactors. IEEE Standard.

IEEEstd1434-2014 (2014). IEEE Guide for the Measurement of Partial Discharges in AC Electric Machinery. IEEE Power and Energy Society and IEEE Dielectrics and Electrical Insulation Society.

Jeyabalan, V. and Usa, S. (2009). Frequency domain correlation technique for pd location in transformer winding. IEEE Transactions on Dielectrics and Electrical Insulation, 19(4), 1160-1167.

Kadlec, P., andfor, B.G., and Strandt, S. (2009). Datadriven soft sensors in the process industry. Computers and Chemical Engineering, 33(4), 795-814.

Kadlec, P., Grbic, R., and Gabrys, B. (2010). Review of adaptation mechanisms for data-driven soft sensors. Computers and Chemical Engineering, 35, 1-24.

Kasabov, N. (1998). The ecos framework and the eco learning method for evolving connectionist systems. Jurnal Adv. Comput. Intell., 2(6), 195-202.

Kasabov, N. (2002). Denfis: Dynamic evolving neuralfuzzy inference system and its application for time-series prediction. IEEE Trans on Fuzzy Syst, 10(2), 144-154.

Kasabov, N. (2007). Evolving connectionist systems: The knowledge engineering approach. Springer Science e Business Media, -.

Khramshin, V.R., Evdokimov, S.A., Nikolaev, A.A., Nikolaev, A.A., and Karandaev, A.S. (2015). Monitoring technical state of the power transformers is a necessary condition of the smart-grid technology introduction within the industrial electric networks. IEEE Young Researchers in Electrical and Electronic Engineering Conference (EIConRusNW), 214-220.

Leite, D., Costa, P., and Gomide, F. (2013). Evolving granular neural networks from fuzzy data streams. Neural Networks, 38, 1-16.

Lin, B., Recke, B., Knudsen, J.K., and Jorgensen, S.B. (2006). A systematic approach for soft sensor development. Computers and Chemical Eng., 31, 419-425.

Lotufo, F.A. and Garcia, C. (2008). Sensores virtuais ou soft sensors: Uma introdução. ra Brazilian Conference on Dynamics, Control and Aplication, 9.

Lucas, F., Costa, P., Batalha, R., and Leite, D. (2018). High impedance fault detection in time-varying distributed generation systems using adaptive neural networks. International Joint Conference on Neural Networks 2018 (IJCNN), 1-8.

Mohamed, R. and Lewin, P.L. (2009). Partial discharge location in high voltage transformers. IEEE Electrical Insulation Conference, -.

Mondal, M. and Kumbhar, G. (2017). A generalized algorithm to compute response of partial discharge in a transformer winding using its equivalent ladder network. 3rd International Conference on Condition Assessment Techniques in Eletrical Systems.

Shao, W., Tian, X., PingWang, Deng, X., and Chen, S. (2015). Online soft sensor design using local partial least squares models with adaptive process state partition. Chemometrics and Intelligent Lab. Syst., 144, 108-121.

Silva, S., Costa, P., Gouvea, M., Lacerda, A., Alves, F., and Leite, D. (2018a). High impedance fault detection in power distribution systems using wavelet transform and evolving neural network. Electric Power Systems Research, 154, 474-483.

Silva, S., Costa, P., Santana, M., and Leite, D. (2018b). Evolving neuro-fuzzy network for real-time high impedance fault detection and classification. Neural Computing and Applications, 1-14.

Skrjanc, I., Iglesias, J., Sanchis, A., Leite, D., Lughofer, E., and Gomide, F. (2019). Evolving fuzzy and neurofuzzy approaches in clustering, regression, identification, and classification: A survey. Information Sciences, 490, 344-368.

Wan, L. and Chan, S. (2016). Pd source diagnosis and localization in industrial high-voltage insulation system via multimodal joint sparse representation. IEEE Transactions on Industrial Electronics, 63(4), 2506-2516.

Watts, M.J. (2009). A decade of kasabov's evolving connectionist systems: A review. IEEE Transactions on Systems, Man, and Cybernetics, 39(3), 253-269.

Yini, L. and Lixing, Z. (2007). A method to locate partial discharge based on the theory of sectional winding transfer functions. The Eighth Int. Conf. on Electronic Measurement and Instruments, 2-493-2-498.

Zhenquan, S., Xuefeng, Z., Jisheng, L., and Yanming, L. (2009). Measurement, diagnostics and monitoring of partial discharges on high-voltage equipment online and off-line. 4th IEEE Conference on Industrial Electronics and Applications, 1521-1526.

Zhu, C., Li, H., and Liu, V. (2015). A novel method of detecting insulation faults in power transformer. International Conference on Smart Grid and Clean Energy Technologies, 116-120. 